\title{
Probabilistic mechanics-based loss scenarios for school buildings in Basel (Switzerland).
}

\author{
C. Michel ${ }^{1), ~}{ }^{*}$, P. Hannewald ${ }^{2)}$, P. Lestuzzi ${ }^{1), 2)}$, D. Fäh ${ }^{2)}$, S. Husen ${ }^{4)}$
}

1) Swiss Seismological Service (SED), Swiss Federal Institute of Technology of Zurich, Switzerland

2) Résonance Ingénieurs-Conseils, Rue Jaques Grosselin 21, 1227 Carouge, Switzerland

3) Ecole Polytechnique Fédérale de Lausanne, EPFL-ENAC-IIC-IMAC, Lausanne, Switzerland

4) Kantonslaboratorium Basel, Kannenfeldstrasse 2, 4012 Basel, Switzerland

Originally published in Bulletin of Earthquake Engineering doi: 10.1007/s10518-016-0025-2

A full-text-view-only version is available at http://rdcu.be/nKYE

\begin{abstract}
Developing earthquake scenarios for cities in areas with a moderate seismicity is a challenge due to the limited amount of available data, which is a source of large uncertainties. This concerns both the seismic hazard, for which only recordings for small earthquakes are available and the unknown earthquake resistance of the majority of structures not designed for seismic loading. The goal of the present study is to develop coherent probabilistic mechanics-based scenarios for a mid-size building stock including a comprehensive analysis of the uncertainties. As an application, a loss assessment for the school buildings of the city of Basel is performed for different scenarios of historical significance, such as the 1356 event, and from the deaggregation of the Swiss Probabilistic Seismic Hazard Model of 2015. The hazard part of the computations (i.e. ground motion estimation) is based on this model, a regional microzonation and recordings of small earthquakes on a dense strong motion network to compute site-amplification factors. The school buildings, which are mainly unreinforced masonry or reinforced concrete shear wall buildings, have been classified according to a specifically developed taxonomy. Fragility curves have been developed using non-linear static procedures and subsequently, vulnerability curves in terms of human and financial losses are proposed. The computations have been run with the OpenQuake engine, carefully propagating all the recognized uncertainties. Scenarios before and after retrofitting measures show their impact on the earthquake safety. A sensitivity analysis shows that the largest uncertainties come from the ground motion prediction although an improvement of all parts of the model is necessary to decrease the uncertainties. Although improved data and models are still necessary to be developed, probabilistic mechanics-based models outperform the capabilities of deterministic and/or empirical models for retrieving realistic earthquake loss distributions.
\end{abstract}

Keywords : Loss assessment, seismic hazard, vulnerability, uncertainty, mechanical, existing buildings

\section{Introduction}

Earthquake scenarios aim at estimating the monetary and human losses for well-defined earthquakes on a portfolio of assets. They are an important tool for decision makers to design appropriate measures to face an event regarding the number of rescue teams, temporary shelters etc. They are also necessary to quantify the human and financial consequences of earthquakes and to evaluate the impact of safety measures such as the retrofitting of buildings.

Most of the earthquake scenarios produced worldwide at the scale of a city are based on empirical methods (i.e. based on macroseismic intensity), which feature a limited level of detail. To overcome the drawback of these methods, mechanics-based loss assessment has 


\section{Conclusions and recommendations for future works}

In this study, loss scenarios have been computed for the city of Basel based on mechanical approaches. A comprehensive estimation of the uncertainties has been performed in order to better understand the sources of uncertainty. The performed scenarios are more precise and robust than previous studies through the coherent combination up to date hazard and vulnerability data and models including their uncertainties. We showed that the latter are large, in the same order of magnitude as the mean values. In the future, the uncertainty in each individual part of the computation has to be decreased to improve the results.

\section{Acknowledgments}

This work has been funded by the Cantonal Crisis Organisation (KKO) of the Canton BaselStadt. The authors thank Laurentiu Danciu who provided the results of the hazard disaggregation. The authors also thank the two anonymous reviewers who helped improve the manuscript.

\section{References}

